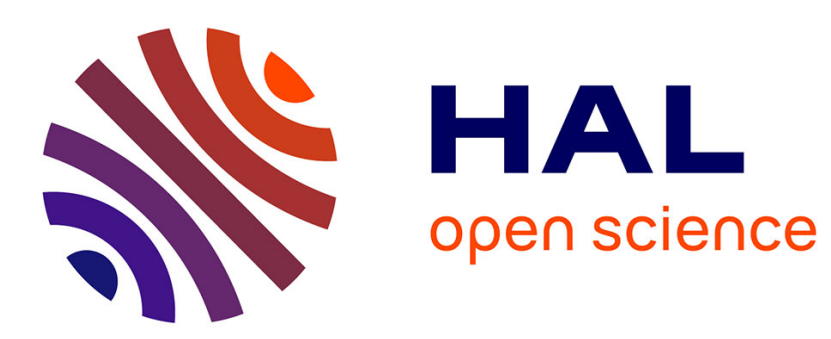

\title{
Leveraging Passive Haptic Feedback in Virtual Environments with the Elastic-Arm Approach
}

Merwan Achibet, Adrien Girard, Maud Marchal, Anatole Lécuyer

\section{To cite this version:}

Merwan Achibet, Adrien Girard, Maud Marchal, Anatole Lécuyer. Leveraging Passive Haptic Feedback in Virtual Environments with the Elastic-Arm Approach. Presence: Teleoperators and Virtual Environments, 2016, 25 (1), pp.17 - 32. 10.1162/PRES_a_00243 . hal-01414830

\author{
HAL Id: hal-01414830 \\ https://hal.inria.fr/hal-01414830
}

Submitted on 12 Dec 2016

HAL is a multi-disciplinary open access archive for the deposit and dissemination of scientific research documents, whether they are published or not. The documents may come from teaching and research institutions in France or abroad, or from public or private research centers.
L'archive ouverte pluridisciplinaire HAL, est destinée au dépôt et à la diffusion de documents scientifiques de niveau recherche, publiés ou non, émanant des établissements d'enseignement et de recherche français ou étrangers, des laboratoires publics ou privés. 


\title{
Leveraging Passive Haptic Feedback in Virtual Environments with the Elastic-Arm Approach
}

\author{
Merwan Achibet \\ Inria/INSA Rennes \\ Adrien Girard \\ Inria Rennes \\ Maud Marchal \\ Inria/INSA Rennes, \\ Corresponding author: maud.marchal@irisa.fr \\ Centre de recherche Inria Rennes - Bretagne Atlantique \\ Campus universitaire de Beaulieu, 35042 Rennes Cedex, France \\ Anatole Lécuyer \\ Inria Rennes
}

This work was partly supported by ANR (MANDARIN project, ANR-12-CORD-0011). 


\begin{abstract}
Haptic feedback is known to improve 3D interaction in virtual environments but current haptic interfaces remain complex and tailored to desktop interaction. In this paper, we describe an alternative approach called "Elastic-Arm" for incorporating haptic feedback in immersive virtual environments in a simple and cost-effective way. The Elastic-Arm is based on a body-mounted elastic armature that links the users hand to his body and generates a progressive egocentric force when extending the arm. A variety of designs can be proposed with multiple links attached to various locations on the body in order to simulate different haptic properties and sensations such as different levels of stiffness, weight lifting, bimanual interaction, etc. Our passive haptic approach can be combined with various 3D interaction techniques and we illustrate the possibilities offered by the Elastic-Arm through several use cases based on well-known techniques such as the Bubble technique, redirected touching, and pseudo-haptics. A user study was conducted which showed the effectiveness of our pseudo-haptic technique as well as the general appreciation of the Elastic-Arm. We believe that the Elastic-Arm could be used in various VR applications which call for mobile haptic feedback or human-scale haptic sensations.
\end{abstract}


Haptic feedback has been shown to improve 3D interaction in virtual environments. Even simple haptic cues, such as notifying about collisions with virtual objects, can significantly improve selection accuracy and task completion time (Pawar \& Steed, 2009; Wall, Paynter, Shillito, Wright, \& Scali, 2002). Haptic effects are also appreciated by users (Vanacken, Raymaekers, \& Coninx, 2006) and increase the "feeling of presence" (Hoffman, Hollander, Schroder, Rousseau, \& Furness, 1998; Insko, 2001).

However, most existing haptic devices have been designed for desktop setups and their workspace is often limited to wrist movements (Iwata, 1990). Several "human-scale" haptic interfaces have been designed for larger workspaces (Dominjon, Perret, \& Lécuyer, 2007) but the resulting hardware is often expensive and cumbersome. Thus, in most cases, haptic devices limit the mobility of the users, which prevents their use in large immersive spaces.

Therefore, in this paper, we propose a simple and cost-effective approach for incorporating haptic feedback in immersive virtual environments. This novel approach, called "Elastic-Arm", is based on an elastic armature that is mounted on the user's body and linked to his hand. In our implementation, we used simple and inexpensive components: consumer-ready arm exercisers, 3D-printed parts, and a tracking system to measure the 3D positions of the two extremities of the elastic link. This setup notably provides an egocentric passive haptic feedback when the user extends the arm with the intent of performing 3D interaction tasks. Such passive force-feedback can be exploited in virtual environments for improving either 3D interaction or the perception of haptic properties of virtual objects.

Our contributions can be summarized as follows:

- the design of a novel body-mounted interface, made of inexpensive components, that provides haptic feedback during 3D interaction with the hand,

- illustrative use cases that expand the workspace of existing interaction techniques originally relying on grounded interfaces; for instance, mobile implementations of the bubble technique for reaching distant object, redirected touching for perceiving collisions with virtual surfaces, and pseudo-haptics for simulating the various levels of stiffness of virtual objects, 
- a pilot user study that assesses the user's appreciation of the Elastic-Arm as well as the effectiveness of the pseudo-haptic effect that we proposed.

In the remainder of this paper, we first present the general concept of the Elastic-Arm and detail its current implementation. Then, we present different use cases that illustrate how this approach can be combined with existing and proven interactive techniques to make them more mobile. Then, the a preliminary user study that evaluates the Elastic-Arm and a pseudo-haptic effect is presented and discussed. Finally, we propose possible extensions of the general concept of the Elastic-Arm to simulate other haptic properties.

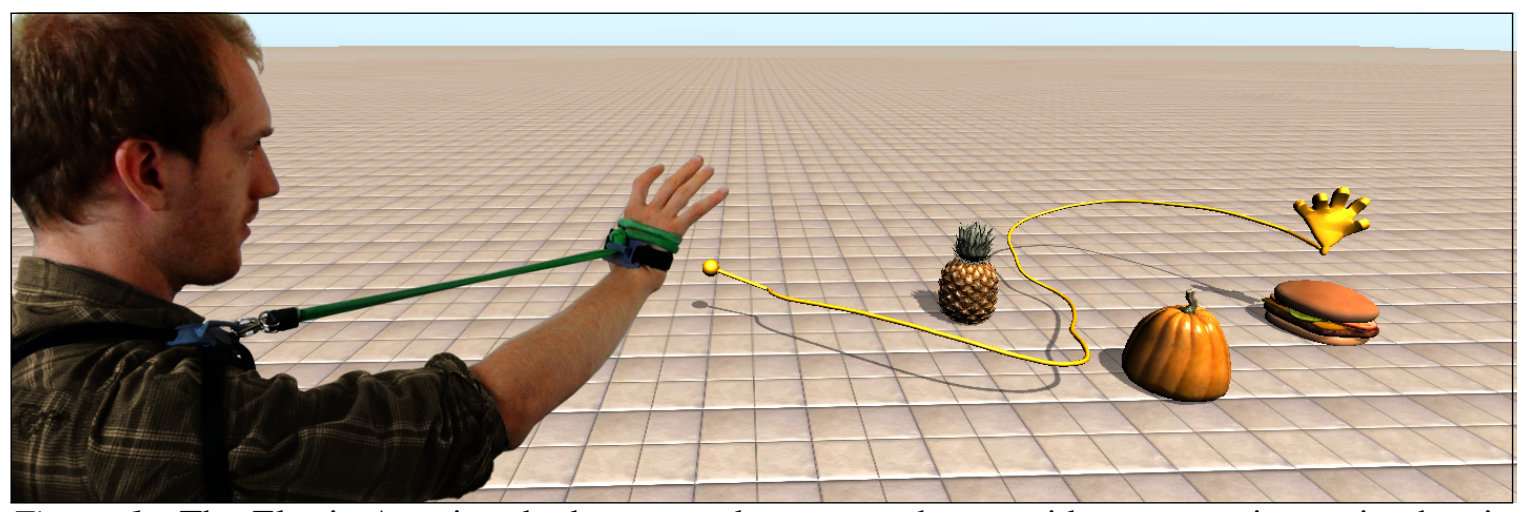

Figure 1. The Elastic-Arm is a body-mounted armature that provides egocentric passive haptic feedback. It presents an alternative to more complex active haptic devices that are generally less adapted to large immersive environments. In this example, the user performs a selection task by stretching his virtual arm using a combination of the Bubble and Go-Go techniques reimplemented with our system.

\section{Related work}

In this section, we describe existing approaches for haptic rendering based either on active or passive interfaces, as well as alternative solutions leveraging cross-modal feedback. We also present the results of studies that investigated the beneficial influence of passive haptic feedback for 3D interaction. 


\section{Active haptic devices}

Haptic devices vary by the degree of mobility that they provide. Desktop devices are the most accessible but they suffer from small workspaces because of their grounded nature and the limited range of their effector (Iwata, 1990). "Human-scale" haptic interfaces have been designed to accompany users within a larger physical space (Dominjon et al., 2007) but they are more bulky and costly. Another option to increase the user's mobility is to directly mount the interface on his body, as is the case for haptic exoskeletons. Such systems are however cumbersome because of their mechanical complexity and can still require to be grounded (Gupta \& O'Malley, 2006).

Simpler string-based interfaces have been proposed to alleviate the issues related to mechanical complexity. For instance, HapticGEAR is a backpack-like device capable of providing force-feedback by pulling on the user's hand with strings (Hirose et al., 2001). Tsetserukou, Sato, and Tachi (2010) proposed a minimalist haptic interface taking the form of an actuated cable that links the wrist to the arm to constrain arm extension, mimicking a muscle. While the degrees of freedom of this system are limited, it can simulate various haptic effects such as weight and collisions. These devices are more accessible thanks to simpler designs but their active nature still induces mechanical overhead and no similar wearable passive haptic devices have been proposed so far.

\section{Passive haptic devices}

Insko (2001) defined passive haptics as : "a technique that incorporates passive physical objects into virtual environments to physically simulate the virtual objects". Therefore, passive interfaces are replicas of virtual objects that provide feedback through their shapes or materials. Examples of such passive feedback include using a physical ledge on the floor to recreate the haptic sensation of standing on the edge of a hole (Insko, 2001) and using a panel made of wood to simulate the tactile feedback of a control panel (Borst \& Volz, 2005). Similarly, Poupyrev, Tomokazu, and Weghorst (1998) used a tangible tablet to emulate the feedback and support of a notepad in a virtual handwriting task.

A second definition is proposed by Swanson (2002): passive haptic devices are "interfaces 
[that] use energetically passive actuators which may in general only remove, store, or redirect kinetic energy within the system". Based on this definition, users are the source of energy and the system can only dissipate or steer that energy. Several haptic devices that fit Swanson's definition have been proposed. For instance, Pihuit, Kry, and Cani (2008) used foam balls as proxies to simulate the grasping of objects and Achibet, Marchal, Argelaguet, and Lécuyer (2014) used hand exercisers. Hoang, Smith, and Thomas (2013) proposed to use a passive deformable glove to perform 3D modeling tasks. Paljic and Coquillart (2004) also developed a passive stringed system with user-actuated brakes to display a stickiness sensation. Through various form factors, these passive devices demonstrated that, even with simple designs, they were able to simulate advanced haptic effects.

\section{Cross-modal alternatives to haptic feedback}

A main issue of passive haptic devices is their low flexibility to support different environments and scenarios, as their feedback cannot be controlled by the simulation. Alternative cross-modal techniques exploit the tight bounds between the visual and haptic modalities in order to provide haptic sensations - or modulate existing ones — through visuals. For example, Kohli (2010) presented redirected touching; a spatial warping that leverages discrepancies between the haptic feedback of the real world and the visual feedback of the virtual world in order to distort shape perception. Similarly, Ban, Kajinami, Narumi, Tanikawa, and Hirose (2012) simulated different shapes by slightly altering the virtual representation of a real prop as well as the position of the user's finger while he follows its outline.

Another solution to extend the possibilities of passive devices is pseudo-haptic feedback (Lécuyer, Coquillart, Kheddar, Richard, \& Coiffet, 2000). It has been initially introduced as a dynamic mismatch between the force applied on an isometric device and the corresponding movement of a virtual object being manipulated (the Control/Display ratio). This effect was illustrated with a task involving the insertion of an object into a duct: when the manipulated object reaches the duct, the movement is slowed down and users increase the force applied on the passive device which makes them perceive a friction force. Pseudo-haptic feedback can also simulate other 
haptic properties such as mass (Dominjon, Lécuyer, Burkhardt, Richard, \& Richir, 2005), torque (Paljic, Burkhardt, \& Coquillart, 2004), and grasping forces (Achibet et al., 2014).

Such cross-modal effects expand the capabilities of passive devices without relying on complex mechanical systems. The approach that we propose in this paper, the Elastic-Arm, is motivated by such effects and aims at providing a similarly convincing feedback with simple and accessible hardware.

\section{Benefits of haptic feedback}

Several user studies investigated the beneficial effects of active force-feedback and showed that it can indeed improve accuracy in selection tasks (Wall et al., 2002) as well as reduce task completion time (Pawar \& Steed, 2009).

The effects of passive haptic feedback have also been specifically investigated. For instance, Hoffman et al. (1998) found that manipulating real props representing virtual objects increased the sense of the presence. Similarly, Insko (2001) investigated the influence of passive haptics in a pit hole scene where feedback was supported by a physical ledge on the floor and showed through physiological measures that passive haptics enhances the sense of presence.

Regarding the performance provided by passive haptics, Lok, Naik, Whitton, and Jr. (2003) found that manipulating physical versions of virtual objects significantly improved completion time. Viciana-Abad, Lecuona, and Poyade (2010) investigated a task consisting in playing with a virtual Simon game and showed a reduction in both execution time and number of errors. Hoang et al. (2013) showed that their passive glove enhanced user's performance and reduced fatigue in a modeling task. Unlike the previous studies, Borst and Volz (2005) compared active and passive feedback in a task consisting of interacting with buttons and sliders on a virtual control panel, physically simulated either with a real tangible surface or with an active haptic device. This experiment showed that there were no significant differences in performance between passive and active feedback for this task. 


\section{The Elastic-Arm: egocentric haptic feedback with passive components}

\section{Concept}

The approach that we propose is based on a novel body-mounted elastic armature that enhances interaction in virtual environments by providing passive haptic feedback to the user's hand. It relies on an elastic cable that links the user's hand to his body (Figure 1). When stretching out the arm in order to perform interaction tasks, an effort proportional to the stiffness of the cable is felt. This egocentric resistance force can then be leveraged in order to incorporate haptic feedback either into interaction techniques without any haptic components or into interaction techniques that originally relied on grounded active devices or passive props. This concept can be generalized furthermore with alternative designs composed of several rubber bands, attached to various locations of the body, in order to simulate different haptic effects, as described in a later section.

\section{Implementation}

For providing the haptic feedback of the Elastic-Arm, elastic cables sold as arm exercisers were used. Each end of the elastic cable is attached through a hook to 3D-printed straps; one on the shoulder and the other on the back of the hand. Velcro bands wrapped around the arm and around the torso ensure that the shoulder strap remains securely fixed when the user extends his arm and pulls the elastic cable (Figure 2(a)).

As illustrated in Figure 2(b), this setup inherently creates a dichotomy between two states: when the elastic is relaxed and when it is taut. The transition between these states can be controlled by the user by extending his arm as well as gauged thanks to the provided haptic feedback. Designing interaction techniques around this feature requires both the position of the hand $\boldsymbol{h}$ and the position of the shoulder $\boldsymbol{s}$ in order to obtain the reach vector $\boldsymbol{r}=\boldsymbol{h}-\boldsymbol{s}$. In our prototype, a pair of Razer Hydra trackers (Sixense, USA) was used for tracking these positions. However, any other tracking system for desktop interaction or for large physical spaces can also be used, camera-based setups for instance. 


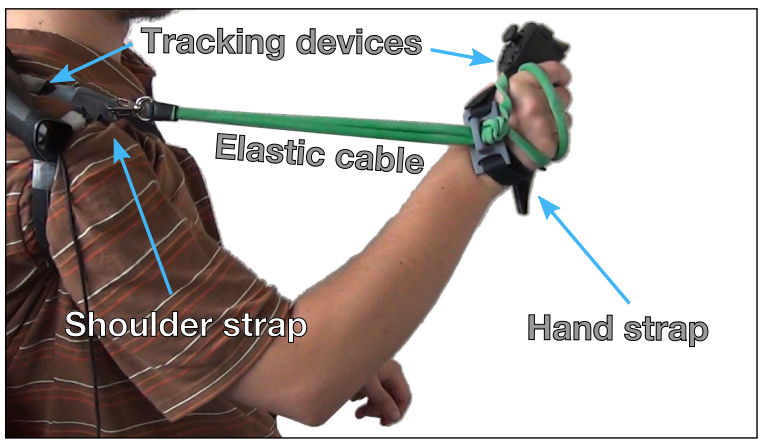

(a)

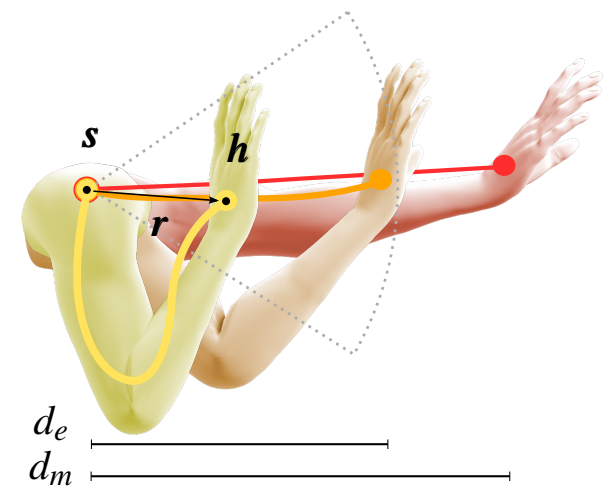

(b)

Figure 2. Elastic armature that constrains the user's hand relatively to his body. (a) It is composed of an elastic cable (an arm exerciser in our prototype), 3D-printed straps, and tracking devices (a Razer Hydra). (b) Different states depending on the extension of the arm: relaxed cable (yellow), nearly taut (orange), and extended arm (red). The dotted radius corresponds to the spherical boundary of the elastic cable beyond which haptic feedback is felt $\left(\|\boldsymbol{r}\|>d_{e}\right)$.

The rest length of the elastic cable is defined as $d_{e}$ (Figure 2(b)). Thus, when the hand is close to the body $\left(\|\boldsymbol{r}\|<d_{e}\right)$, the elastic cable is not taut and the user's arm moves in an unconstrained manner. However, when $\|\boldsymbol{r}\|>d_{e}$, an effort proportional to the stiffness of the cable and the arm extension pulls the arm back. Another potentially useful value is the maximum reach of the user's arm, $d_{m}$. These thresholds can be obtained through a short calibration step by, first, asking the user to stretch his arm until the elastic is taut $\left(d_{e}\right)$ and then asking him to stretch his arm as much as possible $\left(d_{m}\right)$. The thresholds can then be exploited for the design of relevant control schemes.

\section{Discussion}

The design of the Elastic-Arm is motivated by established literature showing that passive haptic feedback is involved in enhancing both performance (Borst \& Volz, 2005; Lok et al., 2003; Viciana-Abad, Reyes-Lecuona, \& Poyade, 2010) and perception (Kohli, 2010; Lécuyer et al., 2000; Paljic et al., 2004). It also provides a stronger sense of presence (Hoffman et al., 1998; Insko, 2001). Through this approach, our aim is to provide a mobile, low-cost, and easily 
reproducible mean to leverage such an essential feedback. Hence, existing interaction techniques could be augmented with the use of our system; which additionally opens the gate for all-new interaction methods specifically designed around its egocentric haptic capabilities.

In order to illustrate the possibilities offered by the Elastic-Arm, the following section presents several application examples, focused on control and perception. For these illustrative use cases, we chose to leverage the virtual hand metaphor since it provides an engaging and meaningful representation for descriptive purposes. This metaphor also fits well with the egocentric nature of the Elastic-Arm. Therefore, some of the demo applications display a bright orange hand (inspired by Mr. Tickle, a cartoon character capable of stretching his arms to perform various feats) and others display an avatar with realistic hands.

\section{Illustrative use cases}

In this section, we present several illustrative use cases based on existing interaction techniques. These techniques were originally designed and evaluated with haptic feedback in mind and rely either on grounded haptic devices or on static passive props. Here, we adapt them to the Elastic-Arm in order to demonstrate how our system can make such methods more mobile whilst providing a similar haptic feedback.

The use cases are divided into two categories. Firstly, we explore how interaction tasks such as object selection and navigation can benefit from the Elastic-Arm. Then, we explore perceptual effects made possible by our system and propose examples of methods for enhancing the users' perception of a virtual environment.

\section{Using the Elastic-Arm to expand the virtual workspace}

First, we propose two illustrative use cases that enable (1) selection of distant objects by stretching a virtual arm and (2) navigation toward out-of-view areas and around obstacles in order to reach occluded objects.

Selection of distant objects with the Bubble technique. The first example that we propose enables users to select virtual objects that are out of reach by stretching their virtual 
arm (Figure 1). This example is based on the hybrid position/rate control of the Bubble technique (Dominjon, Lécuyer, Burkhardt, Andrade-Barroso, \& Richir, 2005). It is also reminiscent of the Go-Go technique with regards to its arm-extension mechanics (Poupyrev, Billinghurst, Weghorst, \& Ichikawa, 1996).

In this scenario, users select objects by touching them with their virtual hand. Similarly to the Bubble technique, two control modes can be differentiated. To begin with, the virtual hand is position-controlled with a 1:1 mapping if $\|\boldsymbol{r}\|<d_{e}$, i.e. when the user performs interaction tasks in his close vicinity. However, when the elastic cable is taut $\left(\|\boldsymbol{r}\| \geq d_{e}\right)$, the virtual hand switches to rate-control and stretches to reach faraway targets (Figure 1). Flexing the arm below the elastic threshold at any moment triggers a rewinding animation that quickly rolls the arm backward to the user's position.

In rate-control mode, when the virtual arm stretches, users have control over the speed of the virtual hand by extending their arm farther away from their shoulder. They also steer the hand by pointing their arm in the direction that they wish to go. These relations are described in the following equation and Figure 3(a), with $\boldsymbol{h}_{\boldsymbol{v}}$ the position of the virtual hand and $k_{p}$ a scaling coefficient:

$$
\boldsymbol{h}_{v}(t+1)=\boldsymbol{h}_{v}(t)+k_{p} \hat{\boldsymbol{r}}\left(\|\boldsymbol{r}\|-d_{e}\right) .
$$

This implementation of the Bubble technique with the Elastic-Arm shares similar principles with its original version that made use of active haptic feedback. Distant targets can similarly be reached by leveraging an hybrid control scheme. However, even without an active device, the interaction is still assisted by the same feedback. Notably, the transition between position and rate control (the boundary of the bubble) can be perceived by users. Similarly, the haptic feedback perceived when stretching the virtual hand is proportional to its speed. As shown by Zhai (1998), rate-control provides better performance with self-centering elastic devices such as ours. Thus, the Elastic-Arm seems well adapted to control schemes like that of the Bubble.

Navigation toward occluded objects with the BubbleCam technique. We propose a variation of the previous method that additionally enables to select occluded objects by navigating 
across the virtual environment and around obstacles. The associated controls are similar to the previous method but the displayed view is similar to the BubbleCam technique (Dominjon, Lécuyer, Burkhardt, Andrade-Barroso, \& Richir, 2005).

Here, the virtual camera displaying the scene is fixed to the virtual hand. In this way, the precision of the control does not decrease as the hand moves away since the user keeps the same relative viewpoint. In order to accommodate the egocentric nature of the Elastic-Arm, we also complemented the Bubble's original control scheme with rotations: users can rotate their virtual hand by pointing away from the forward direction $\boldsymbol{F}$. These relations are summed up by the following equations and Figure 3(a), with $\boldsymbol{h}_{\boldsymbol{v}}$ and $o_{v}$ the position and orientation of the virtual hand, $k_{p}$ and $k_{o}$ scaling coefficients and $q$ the quaternion representing the rotation from $\boldsymbol{F}$ to $\boldsymbol{r}$ :

$$
\boldsymbol{h}_{v}(t+1)=\boldsymbol{h}_{v}(t)+k_{p} \hat{\boldsymbol{F}}\left(\|\boldsymbol{r}\|-d_{e}\right), \quad \boldsymbol{o}_{v}(t+1)=\boldsymbol{o}_{v}(t) q^{k_{o}}
$$

The combination of a fixed camera and rotational controls enables to navigate along more intricate paths in order to reach occluded objects. For instance, Figure 3(b) illustrates how an object hidden behind a wall can be selected. In order to provide true navigation capabilities, users are also able to set the current position of the hand as a new starting position by pressing a button (the trigger of the Razer Hydra in our prototype). In this way, they can navigate to different points of interest and then stretch their arm to grab objects before rolling back to the chosen position.

\section{Using the Elastic-Arm to improve perception}

The second set of illustrative use cases that we propose explores how the Elastic-Arm can improve the perception of the virtual environment. Two different examples are proposed: (1) a redirection effect to touch the virtual environment through our elastic armature and (2) a pseudo-haptic effect that allows users to perceive varying levels of effort when interacting with virtual objects.

Perception of virtual boundaries with redirected touching. For this illustrative use case, our aim is to provide users with haptic cues that relate to the physical bounds of the virtual environment so that they can perceive its limits by probing their vicinity. This example is inspired 


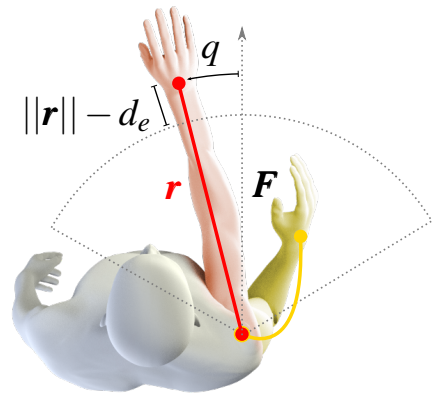

(a)
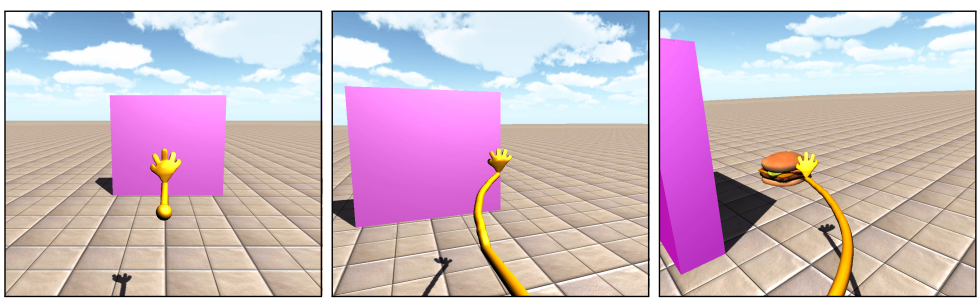

(b)

Figure 3. Control scheme for the stretchable arm. (a) The control mode depends on the extension of the arm: the virtual hand is position-controlled below the elastic threshold (yellow) and the hand is rate-controlled beyond the elastic threshold (red). (b) Successive frames of the user's view when navigating around obstacles to reach an occluded object. The camera follows the virtual hand to maintain a constant level of precision.

by redirected touching (Kohli, 2010), an illusory effect that leverages a passive prop and alters its virtual appearance as well as the position of the users' virtual hands on its outline to make them perceive different shapes when they touch it. Similarly, the method proposed here relies on a discrepancy between visual and real positions: we alter the position of the users' virtual hand so that its encounter with an obstacle is correlated with the elastic cable being taut. In this way, users are able to perceive a clear resistance when "touching" virtual objects. Since this effect relies on a visual discrepancy, users wear a head-mounted display so that only their virtual hand is visible.

The alteration of the virtual hand position consists in varying its Control/Display ratio with respect to the distance to facing obstables. In order to obtain this distance $d_{o}$, rays are continuously cast in the $\boldsymbol{r}$ direction. The Control/Display ratio between real and virtual hands is then adjusted so that the cable tension matches the potential collision with the obstacle, as illustrated in Figures 4(a) and 4(b). In other words, the distance to the obstacle is mapped on the rest length of the elastic cable. The following equation sums up this principle ( $\boldsymbol{s}$ is the shoulder position):

$$
\boldsymbol{h}_{v}(t)=\boldsymbol{s}+\hat{\boldsymbol{r}} d_{o} \min \left(1, \frac{\|\boldsymbol{r}\|}{d_{e}}\right)
$$


Figure 4(c) presents a scene featuring this redirection effect. Users are seated in a control room, the parts of which they can touch through the Elastic-Arm. This application of the idea of redirected touching with the Elastic-Arm enables to feel virtual obstacles that are within the user's reach. However, contrary to the original implementation, users do not have to stay in front of a grounded passive prop since the haptic feedback is provided by the body-mounted armature. This technique could thus make a large virtual environment tangible.

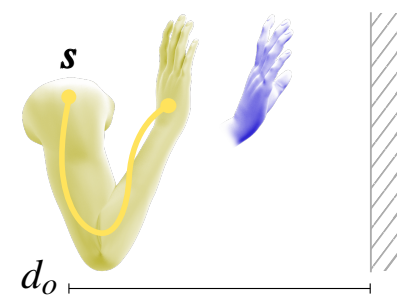
(a)

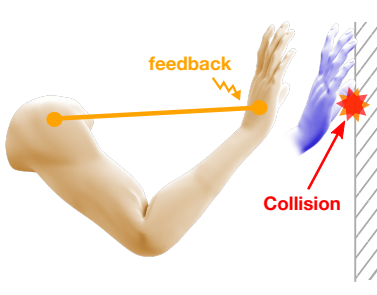

(b)

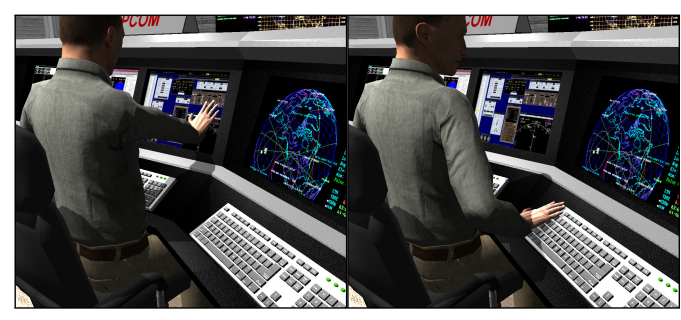

(c)

Figure 4. Simulating virtual boundaries with the Elastic-Arm. (a) The user's arm is below the elastic threshold and the virtual hand (blue) does not collide with any obstacle yet; there is no feedback. (b) The virtual hand now collides with an obstacle. The Control/Display ratio has been adjusted to match this event with the tension of the elastic cable and a resistance is felt. (c) Users can explore virtual environments and "touch" virtual objects at various distances, like screens or the keyboard.

Perception of variable levels of effort with pseudo-haptics. The second perception-oriented use case that we present is based on pseudo-haptic feedback (Lécuyer et al., 2000) and leverages the Elastic-Arm to simulate different levels of effort when interacting with virtual objects. Pseudo-haptics is an alternative means of delivering haptic sensations that simulates haptic properties by relying on visual feedback coupled with the actions of the user. Here, we apply this principle and alter the speed of the user's virtual hand depending on the haptic properties of the object it is interacting with. In this way, users have to stretch their arm to different degrees depending on the object. In consequence, they perceive different levels of effort thanks to the elastic nature of our armature.

This effect builds on the previous technique based on redirected touching, since the virtual hand must first collide with an object and the elastic cable must be just taut in order to start the 


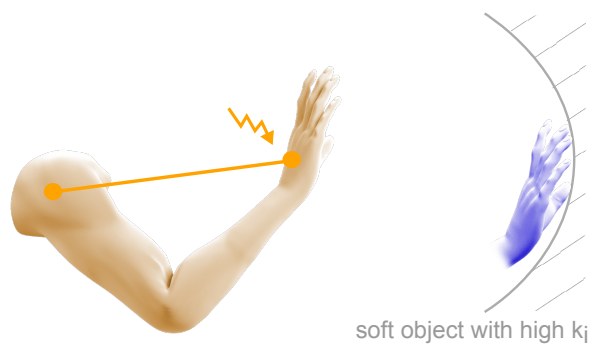

(a)

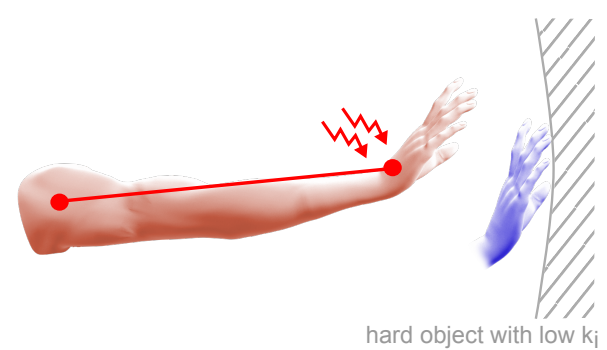

(b)

Figure 5. Simulating different levels of effort with the Elastic-Arm. Two different deformable objects are interacted with. (a) The object is soft so the hand motion is amplified and the user moderately stretches his arm to bend the surface; the haptic feedback is moderate. (b) The object is stiff so the hand motion is slowed down and the user must stretch his arm to a greater degree to reach the same amount of deformation; the haptic feedback is stronger.

effect. Then, once the interaction begins, the motion of the virtual hand is altered depending of the object properties. In practice, each object is associated with a different interaction coefficient $k_{i}$ that governs how the motion of the virtual hand is scaled during the interaction $\left(k_{i} \in[0,1[\right.$ slows down the motion, $\left.k_{i} \in\right] 1, \infty[$ amplifies it). The following equation describes this principle:

$$
\boldsymbol{h}_{\boldsymbol{v}}(t)=\boldsymbol{s}+\hat{\boldsymbol{r}} d_{o} \min \left(1, \frac{\|\boldsymbol{r}\|}{d_{e}}\right)+\hat{\boldsymbol{r}} k_{i} \frac{\|\boldsymbol{r}\|-d_{e}}{d_{m}-d_{e}}
$$

Through this effect, users can interact with different objects and distinguish various levels of effort. In Figure 5, two deformable objects are pushed on and the one with the smaller $k_{i}$ bends to a lesser degree, even though the arm is stretched more, and the resulting haptic feedback is greater. Figure 6 shows a demonstration application in which users can push two different buttons and perceive different effort requirements to activate them. More generally, this effect could simulate various haptic properties related to pushing actions, such as closing a drawer or pushing a wheeled cart.

\section{User evaluation: distinguishing virtual buttons with different stiffness}

We conducted a preliminary user study in order to verify that users could indeed perceive different levels of effort through the pseudo-haptic effect presented in the previous section. We 


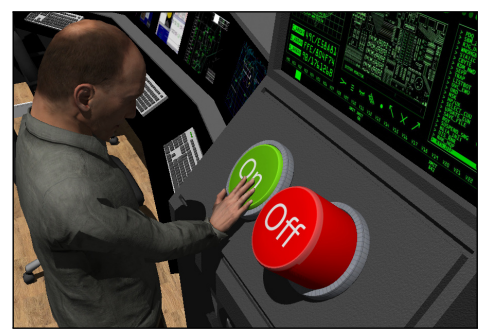

(a)

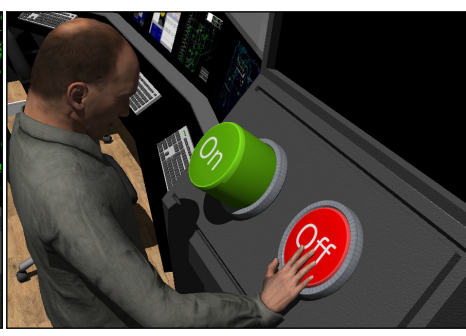

(b)

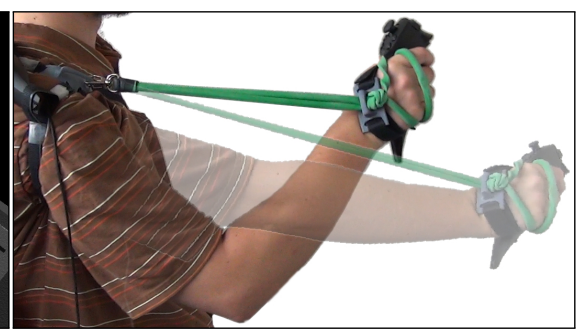

(c)

Figure 6. Pseudo-haptic effect simulating different levels of effort. (a) The button on the left is associated to an interaction coefficient $k_{i}=1$ so the motion of the user's hand is unaltered when pushing it. (b) The button on the right is associated to a lower interaction coefficient $k_{j}<k_{i}$ so the motion of the user's hand is slowed down. (c) User's posture when pushing on the first button. For pushing the stiffer button, the user compensates the speed difference by extending the arm furthermore (faded arm), which generates stronger haptic cues.

thus asked participants to complete a stiffness discrimination task in which they had to interact with a collection of virtual buttons and to sort them according to their level of stiffness.

Apparatus and participants. The environment of the experiment was displayed through an Oculus Rift head-mounted display (Oculus VR, USA). Participants were embodied by a virtual avatar and the virtual camera was placed at the level of their eyes. The avatar was seated with the same position as participants and they could freely look around the environment.

In one experimental condition, participants wore the elastic armature. The positions of their shoulder and their dominant-hand were tracked in space with a pair of Razer Hydra sensors. They could move the right arm of the avatar by moving their own arm with a 1:1 mapping (except when the pseudo-haptic effect was enabled). The input devices and head-mounted display were calibrated for each participant at the beginning of the experiment.

Eight participants took part in the experiment; six were male and two were female, aged from 22 to $31(M=24.6, S D=6.2)$. Seven of them were right-handed and one was left-handed.

Procedure. Participants were asked to sort virtual buttons according to the amount of effort that they required to push on. Buttons were placed in front of the participants one at a time (Figure 7(a)) and they were identified by prominent bold letters (A, B, or C). To ensure a 
consistent amount of interaction for each button, participants had to push the current button to at least $80 \%$ of its course, which switched on a red light as a visual cue. Then, participants were asked to release the button and bend the arm to display the next button. Once the three buttons had been interacted with, participants had to select which one they considered as requiring the most effort to push. Three floating letters appeared in front of the users and they chose an answer by placing their hand on the corresponding letter (Figure 7(b)). Then the selected answer disappeared and participants had to select the button that required the least effort to push among the remaining options. Finally, participants were asked to return to their initial position, with the arm close to the body, to trigger the next trial.

Conditions. This preliminary user study has different goals. First, it aims at gathering subjective input from users about their experience with the Elastic-Arm. Secondly, its aims at assessing if users do perceive different levels of effort when interacting through the elastic armature, with the proposed pseudo-haptic effect.

For this reason, participants are subjected to the two following conditions. In the WITH condition, they wear the Elastic-Arm to constrain their hand during the interaction. In the WITHOUT condition, users interact without wearing the elastic armature. In both conditions, participants control their avatar to interact with the buttons in the same manner, by moving their own arm in space. Likewise, the pseudo-haptic effect that simulates different levels of effort is always enabled and uses the same parameters. The only difference is the addition of passive egocentric haptic feedback. Hence, these conditions will help verify that the different levels of effort perceived by participants are not only due to the visual feedback (altered speed of the hand when pushing the buttons) but also to the contribution of our elastic armature.

Experimental design and collected data. The independent variables of the experiment were the CONDition (With, Without), the ORDERING of the three buttons, and the

\section{REPETITION.}

The buttons were associated to three different interaction coefficients that governed the pseudo-haptic effect: $k_{\text {low }}=0.5, k_{\text {mid }}=1, k_{\text {high }}=2$. The attribution of these coefficients was counterbalanced across all trials. The answers of participants when sorting the buttons is the main 
measure. We consider an answer as valid if the interaction coefficients of the corresponding buttons are ordered in the correct order.

We additionally asked participants to fill a subjective questionnaire to rate each condition in terms of appreciation, ease of use, accuracy, comfort, fatigue, as well as their perception of of the task on a 5-Likert scale. The questions are listed in Figure 7(c). A score of 5 for these criteria means a very positive rating except for fatigue where it means that the technique was very tiring (a rating of 1 means that it was not tiring at all). The participants were also asked to indicate which condition they preferred: visual feedback only (WITHOUT condition) or both haptic and visual feedback (WITH).

The total duration of the experiment was approximately 15 minutes. In total, we had 8 participants $\times 2$ conditions $\times 6$ combinations $\times 2$ repetitions $=192$ trials.

Results. Ordering of the virtual buttons - Regarding the answers of participants, we performed a generalized likelihood test and we found a significant difference between the two conditions $\left(\chi^{2}=10.24, p=0.001\right)$. The orderings were more often correct when using the WITH condition, with a 94\% probability, whereas they were correct with the WITHOUT condition with a probability of $80 \%$.

Subjective questionnaire - Regarding the subjective answers of the participants, a t-test showed no significant effect for the appreciation, ease of use, and accuracy criteria (Figure 7(c)). We found a significant effect for the comfort criterion in favor of the WITHOUT condition (With $=3.625$, Without $=4.5, p=0.041)$ as well as for the fatigue criterion $($ With $=3.125$, Without $=2.375, p=0.019)$. There was also a significant effect for the perception of different levels of effort criterion in favor of the WITH condition (With $=4.875$, Without $=3.875, p=0.049$ ). Regarding user's preferences, two participants declared that they preferred the WITHOUT condition and six participants preferred the WITH condition.

Discussion. The preliminary evaluation gave insights about the appreciation of the Elastic-Arm by users as well as the effectiveness of the pseudo-haptic effect that we proposed. First, the subjective questionnaire revealed that participants rated our armature as less comfortable and more tiring that the unconstrained condition. This opinion is reflected in some 

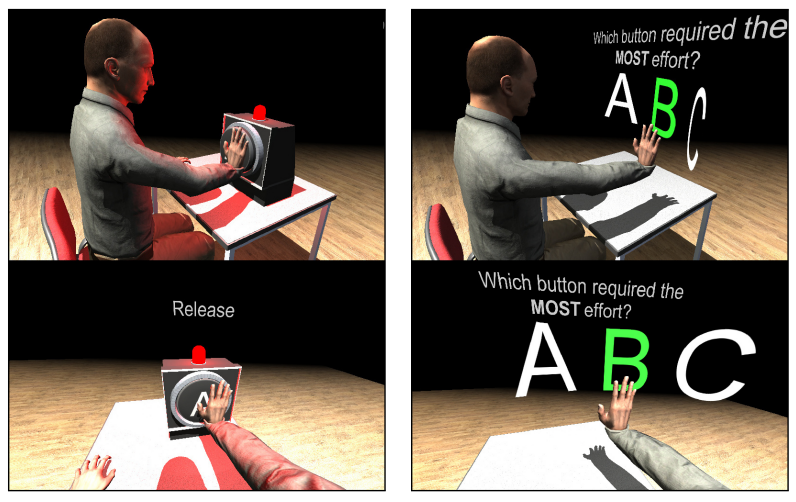

(b)

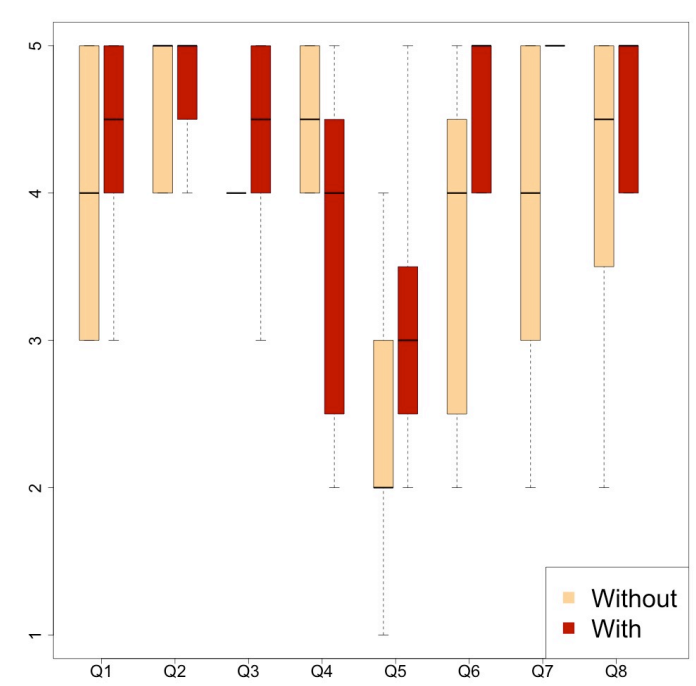

(c)

Figure 7. (a, b) Different steps of the experimental procedure (top: external perspective, bottom: participant's view). (c) Answers to the subjective questionnaire on a 5-Likert scale (the whiskers represent the range of answers). Q1: You liked this technique. Q2: This technique was easy to use. Q3: This technique was accurate. Q4: This technique was comfortable. Q5: This technique was tiring. Q6: The buttons required some effort to push. Q7: The buttons required different levels of effort to push. Q8: You were confident about your answers.

comments from participants: "[It was] a bit tiring at the end of the experiment", "I prefer not to get tired by moving the arm that much". Those results were somehow expected from such modes of interaction that engage users physically. Thus, in order to reduce fatigue, applications making use of the Elastic-Arm should carefully assess the range and frequency of movements that users would have to perform.

However, the Elastic-Arm showed to provide a richer understanding of the virtual environment than simple "in-the-air" unconstrained interaction. Indeed, participants provided correct orderings significantly more often when they were equipped with our armature, and they also rated the Elastic-Arm as being significantly better to distinguish the stiffness of the virtual buttons. Those elements suggest that the association of elastic armature and pseudo-haptic effect do provide an advantage in terms of sensations, that cannot be provided by visual feedback alone. 
Additionally, some users mentioned in the questionnaire that they appreciated that the Elastic-Arm provides a sense of tangibility to the virtual environment: "It really feels like pushing a button", "This is more realistic". Finally, participants predominantly chose the Elastic-Arm as their favorite technique.

\section{Alternative designs for simulating new haptic properties}

In this section, we present alternative setups using the Elastic-Arm concept to simulate other haptic sensations. The first extension explores how to physically provide several levels of effort with a multi-string armature. The second extension illustrates how additional haptic effects can be enabled by considering the waist as another anchor point for the rubber band to provide downwards forces and simulate weight.

\section{Multi-layer rendering with several rubber bands}

With the current prototype of the Elastic-Arm, the magnitude of the haptic feedback is limited to the stiffness of the rubber band that is used. However, more elaborate rigs with several elastic cables of different lengths could be attached on the same hand. In this way, more "layers" of stiffness could divide the user's reach.

This technique generalizes the redirected touching technique previously presented by synchronizing the tension of each rubber band with one change of stiffness. For example, the "button pushing" task that we evaluated could be enriched if the shortest elastic was just tightened when the user hand touches the surface of the button (Figure 8(b)), and the longer rubber band (or a rigid string) is tightened when the button meets the end of its course (Figure 8(c)).

This multi-string armature progressively accumulates the stiffness of several rubber bands, and could thus be used to simulate virtual objects with complex material layers (e.g. a soft layer followed by a stiffer one), or interaction in heterogeneous environments. An example of game-like scenario using this type of feedback would be a task in a basin of water with different levels of density. For instance, users would start above the basin (no stiffness), immerse their hand in the water (low stiffness), reach the sand at the floor to find buried objects (medium stiffness), and 
finally hit the glass at the bottom (high stiffness).

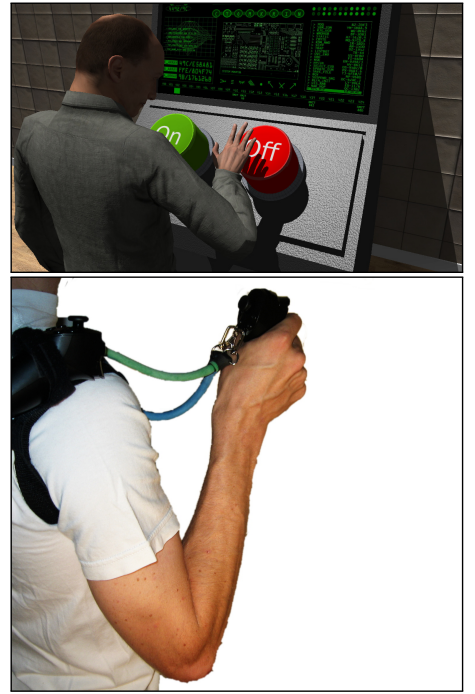

(a)

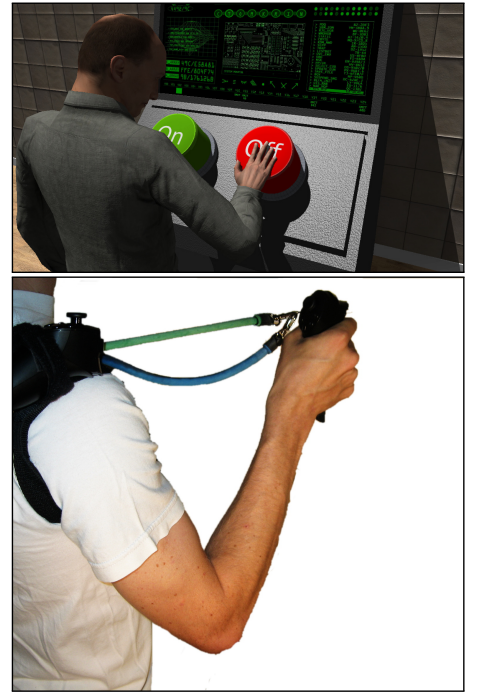

(b)

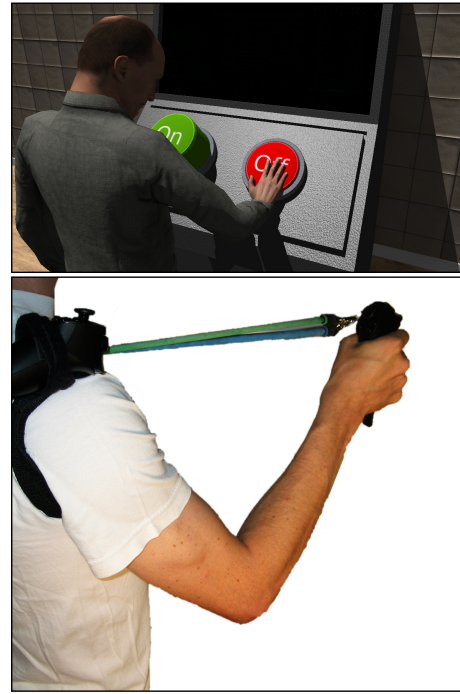

(c)

Figure 8. Multi-layer rendering for providing different levels of stiffness. (a) The elastic is not taut when there is no collisions with the virtual hand. (b) The shortest elastic is tightened just when the hand touches the virtual button. (c) The second and longest elastic is tightened when the button reaches the end of its course.

\section{Weight simulation from the waist}

Currently, the haptic feedback provided by the Elastic-Arm is unidirectional, in the sense that the armature always pulls the user's hand toward his shoulder. Specific haptic properties could be provided by considering alternative anchor points for the elastic cables. Here, we illustrate how to simulate weight by attaching the rubber cable to the the waist and altering the vertical motion of a manipulated virtual object. This extension is inspired by an early work on pseudo-haptic feedback, which simulated different weights by only altering the vertical motion of a sphere controlled through a haptic interface (Dominjon, Lécuyer, Burkhardt, Richard, \& Richir, 2005). Recent work also focused on the simulation of mass through alterations of the visual feedback; for instance Ban et al. (2013) changed the color of virtual dumbbells to change the perceived endurance during a lifting task and Jáuregui et al. (2014) altered the lifting animation of virtual 
avatars to express various weights.

In the implementation that we propose, the rubber band pulls the user's hand downward when he lifts the arm, which provides a first haptic feedback. Then, a pseudo-haptic effect modulates this sensation by scaling the vertical motion of the hand, depending on the weight of the manipulated object. For example, in Figure 9, a user interacts with two different virtual dumbbells. The motion of the lighter dumbbell (Figure 9(a)) is mapped on the user's real motion. However, the motion of the heavier dumbbell (Figure 9(b)) is scaled down vertically, and users have to raise their real hand higher to reach the same position in the virtual environment, which increases the intensity of the downward force.

In a second example, we consider both hands, linked to the waist with separate rubber bands. Each hand is associated to a different Control/Display ratio in order to simulate an asymmetric weight distribution in large objects held with both hands. For instance, in Figure 9(c), weights on the right side of the user are heavier, thus the motion of the right virtual hand is slowed down. The user keeps the barbell straight by raising his right hand to compensate this difference, which provides stronger haptic cues on the heavier side.

\section{Discussion}

The first illustrative use cases that we presented explored how the Elastic-Arm could apply to proven interaction techniques, both in terms of control and in terms of perception. Then alternative designs of the Elastic-Arm concept illustrated how it could simulate other haptic properties such as layered levels of stiffness and weight. Future work could consist in designing full-fledged interaction techniques from the ideas explored in this paper and then assess their effect on performance and perception in specific application contexts. We could especially investigate the possibilities offered by the Elastic-Arm when used in large physical spaces.

Indeed, compared to desktop haptic devices with limited clearance, the Elastic-Arm provides an extended workspace. Since, it is always centered on the user's shoulder, the workspace is by design as large as the user's reach. Moreover, unlike with desktop devices or static passive props, users do not have to keep the same fixed position and they are free to move around a larger 
physical space such as an immersive CAVE.

Another convenience of the Elastic-Arm is its simple and reproducible design. Its components (elastic cable, 3D-printed straps) make it easy to build. The elastic cable can be found in a variety of lengths to accommodate different user morphologies. Similarly, it can be found with different levels of stiffness depending on the targeted application context. This makes the Elastic-Arm an affordable and versatile system that could find uses in many domains.

We could consider equipping more body parts with similar elastic rigs. For instance, fingers could be connected by short elastic cables in order to design new manipulation techniques enhanced by a portable passive feedback. Additionally, new setups could be designed to handle both hands and constrain their relative positions when conducting bimanual tasks like manipulating virtual objects. While the hand is a major tool for 3D interaction, other body parts could be considered too and another possibility would be to equip the legs with similar elastic armatures. In this specific instance, novel navigation techniques based on the provided haptic feedback could be designed. From a more general perspective, designing such localized elastic armatures could lead to the design of an "elastic exoskeleton" and make general-purpose haptic feedback more accessible for Virtual Reality applications.

\section{Conclusion}

In this paper, we proposed the Elastic-Arm, a body-mounted armature providing passive haptic feedback to the user's hand. The simple design of our system enables haptic feedback within a large workspace compared to more complex and more costly active haptic devices and compared to static passive props. Several illustrative use cases were presented to illustrate the capabilities of our system, focusing either on expanding the user's reach in virtual environments or enriching his perceptions.

A preliminary user study evaluated a pseudo-haptic effect by exposing users to a stiffness discrimination task in which they had to order virtual buttons. Results showed that participants answered correctly significantly more often when wearing the Elastic-Arm, and that they predominantly preferred to use the elastic armature compared to free, unconstrained interaction. 
This work leads us to believe that the Elastic-Arm could be leveraged for a wide range of tasks and haptic properties, as illustrated with a multi-string setup that simulates layers of stiffness and by a waist-based design for simulating weight. It could also be incorporated in a wide range of contexts. For example, we can also envision useful uses of such systems for ergonomics studies, video games, or sport training. Another field of application that would fit the Elastic-Arm is medical rehabilitation since tasks of increasing difficulty could be proposed to patients in order to progressively enhance their physical performance, either by equipping them with armature with increasing levels of stiffness or by leveraging our pseudo-haptic approach.

\section{References}

Achibet, M., Marchal, M., Argelaguet, F., \& Lécuyer, A. (2014). The virtual mitten: A novel interaction paradigm for visuo-haptic manipulation of objects using grip force. In Proc. of Symposium on $3 D$ User Interfaces (pp. 59-66).

Ban, Y., Kajinami, T., Narumi, T., Tanikawa, T., \& Hirose, M. (2012). Modifying an identified angle of edged shapes using pseudo-haptic effects. In Proc. of EuroHaptics (p. 25-36).

Ban, Y., Narumi, T., Fujii, T., Sakurai, S., Imura, J., Tanikawa, T., \& Hirose, M. (2013). Augmented endurance: controlling fatigue while handling objects by affecting weight perception using augmented reality. In Proc. of Conference on Human Factors in Computing Systems (pp. 69-78).

Borst, C. W., \& Volz, R. A. (2005). Evaluation of a Haptic Mixed Reality System for Interactions with a Virtual Control Panel. Presence, 14(6), 677-696.

Dominjon, L., Lécuyer, A., Burkhardt, J.-M., Andrade-Barroso, G., \& Richir, S. (2005). The "bubble" technique: Interacting with large virtual environments using haptic devices with limited workspace. In proc. of Eurohaptics Conference (p. 639-640).

Dominjon, L., Lécuyer, A., Burkhardt, J.-M., Richard, P., \& Richir, S. (2005). Influence of control/display ratio on the perception of mass of manipulated objects in virtual environments. In Proc. of IEEE Virtual Reality (p. 19-25).

Dominjon, L., Perret, J., \& Lécuyer, A. (2007). Novel Devices and Interaction Techniques for Human-scale Haptics. The Visual Computer, 23(4), 257-266.

Gupta, A., \& O'Malley, M. (2006). Design of a Haptic Arm Exoskeleton for Training and Rehabilitation. IEEE/ASME Transactions on Mechatronics, 11(3), 280-289. 
Hirose, M., Hirota, K., Ogi, T., Yano, H., Kakehi, N., Saito, M., \& Nakashige, M. (2001). HapticGEAR: the development of a wearable force display system for immersive projection displays. In Proc. of IEEE Virtual Reality (pp. 123-129).

Hoang, T. N., Smith, R. T., \& Thomas, B. H. (2013). 3D interactions with a passive deformable haptic glove. In Proc. of International Symposium on Mixed and Augmented Reality (p. 1-6).

Hoffman, H. G., Hollander, A., Schroder, K., Rousseau, S., \& Furness, T. (1998). Physically Touching and Tasting Virtual Objects Enhances the Realism of Virtual Experiences. Virtual Reality, 3(4), 226-234.

Insko, B. E. (2001). Passive haptics significantly enhances virtual environments (Unpublished doctoral dissertation). The University of North Carolina.

Iwata, H. (1990). Artificial reality with force-feedback: development of desktop virtual space with compact master manipulator. In Proc. of ACM SIGGRAPH (pp. 165-170).

Jáuregui, D. A. G., Argelaguet, F., Olivier, A., Marchal, M., Multon, F., \& Lécuyer, A. (2014). Toward "Pseudo-Haptic Avatars": Modifying the Visual Animation of Self-Avatar Can Simulate the Perception of Weight Lifting. IEEE Transactions on Visualization and Computer Graphics, 20(4), $654-661$.

Kohli, L. (2010). Redirected touching: Warping space to remap passive haptics. In Proc. of IEEE Symposium on 3D User Interfaces (p. 129-130).

Lécuyer, A., Coquillart, S., Kheddar, A., Richard, P., \& Coiffet, P. (2000). Pseudo-haptic feedback: Can isometric input devices simulate force feedback? In Proc. of IEEE Virtual Reality (p. 83-90).

Lok, B., Naik, S., Whitton, M. C., \& Jr., F. P. B. (2003). Effects of handling real objects and avatar fidelity on cognitive task performance in virtual environments. In Proc. of IEEE Virtual Reality (pp. 125-132).

Paljic, A., Burkhardt, J.-M., \& Coquillart, S. (2004). Evaluation of pseudo-haptic feedback for simulating torque: A comparison between isometric and elastic input devices. In Proc. of International Symposium on Haptic Interfaces for Virtual Environment and Teleoperator Systems (p. 216-223).

Paljic, A., \& Coquillart, S. (2004). A passive stringed haptic system for immersive environments. In Proc. of EuroHaptics (p. 82-87).

Pawar, V., \& Steed, A. (2009). Evaluating the influence of haptic force-feedback on 3D selection tasks using natural egocentric gestures. In Proc. of IEEE Virtual Reality (p. 11-18).

Pihuit, A., Kry, P., \& Cani, M.-P. (2008, June). Hands on virtual clay. In Proc. of IEEE International Conference on Shape Modeling and Applications (p. 267-268).

Poupyrev, I., Billinghurst, M., Weghorst, S., \& Ichikawa, T. (1996). The Go-Go interaction technique: 
Non-linear mapping for direct manipulation in VR. In Proc. of Symposium on User Interface Software and Technology (p. 79-80).

Poupyrev, I., Tomokazu, N., \& Weghorst, S. (1998). Virtual notepad: handwriting in immersive VR. In Proc. of Virtual Reality Annual International Symposium (pp. 126-132).

Swanson, D. K. (2002). Implementation of arbitrary path constraints using dissipative passive haptic displays (Unpublished doctoral dissertation). School of Mechanical Engineering, Georgia Institute of Technology,.

Tsetserukou, D., Sato, K., \& Tachi, S. (2010). Exointerfaces: Novel exosceleton haptic interfaces for virtual reality, augmented sport and rehabilitation. In Proc. of Augmented Human International Conference (pp. 1-6).

Vanacken, L., Raymaekers, C., \& Coninx, K. (2006). Evaluating the influence of multimodal feedback on egocentric selection metaphors in virtual environments. In Proc. of Haptic and Audio Interaction Design (p. 12-23).

Viciana-Abad, R., Reyes-Lecuona, A., \& Poyade, M. (2010). The Influence of Passive Haptic Feedback and Difference Interaction Metaphors on Presence and Task Performance. Presence, 19(3), 197-212.

Viciana-Abad, R., Lecuona, A. R., \& Poyade, M. (2010, June). The Influence of Passive Haptic Feedback and Difference Interaction Metaphors on Presence and Task Performance. Presence, 19(3), 197-212.

Wall, S. A., Paynter, K., Shillito, A. M., Wright, M., \& Scali, S. (2002). The effect of haptic feedback and stereo graphics in a 3D target acquisition task. In Proc. of Eurohaptics.

Zhai, S. (1998, November). User performance in relation to 3D input device design. ACM SIGGRAPH Computer Graphics, 32(4), 50-54. 


\section{List of Figures}

1 The Elastic-Arm is a body-mounted armature that provides egocentric passive haptic feedback. . . . . . . . . . . . . . . . . . . 4

2 Elastic armature that constrains the user's hand relatively to his body . . . . . . . 9 9

3 Control scheme for the stretchable arm. . . . . . . . . . . . . . . . . . 12

4 Simulating virtual boundaries with the Elastic-Arm . . . . . . . . . . . . . . 13

5 Simulating different levels of effort with the Elastic-Arm . . . . . . . . . . 15

6 Pseudo-haptic effect simulating different levels of effort . . . . . . . . . . . . . 16

7 Different steps of the experimental procedure. . . . . . . . . . . 18

8 Multi-layer rendering for providing different levels of stiffness $\ldots \ldots \ldots$

9 Simulating weight with rubber bands anchored on the waist. . . . . . . . . . 28 


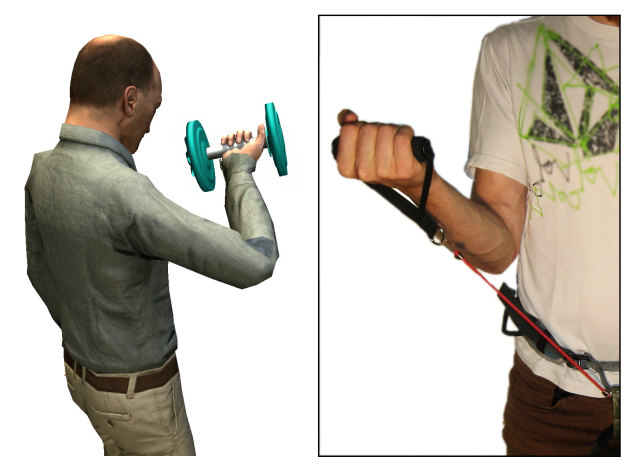

(a)

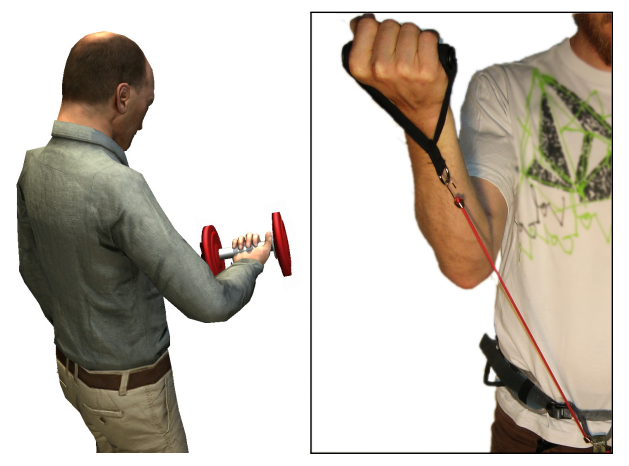

(b)

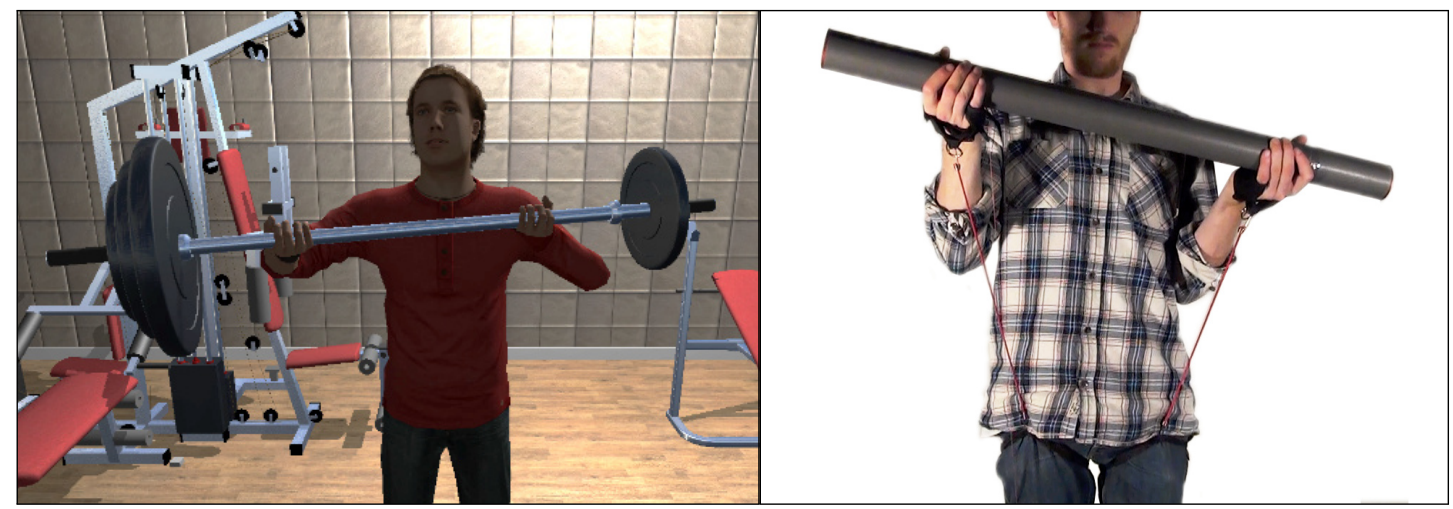

(c)

Figure 9. Simulating weight with rubber bands anchored on the waist. (a) Lighter dumbbell associated to a neutral Control/display ratio. (b) Heavier dumbbell associated to a lower ratio. To lift it, the user has to raise his arm higher, which extends the rubber band furthermore and produces stronger downward forces. (c) Simulating imbalance by scaling the vertical motion of each hand asymmetrically. The user compensates this difference to keep the barbell straight, which generates stronger downward forces on the heavier side. 